\title{
METHIONINE ADENOSYLTRANSFERASE AS A USEFUL MOLECULAR SYSTEMATICS TOOL REVEALED BY PHYLOGENETIC AND STRUCTURAL ANALYSES
}

Gabino F. Sánchez-Pérez ${ }^{1}$, José M. Bautista ${ }^{2}$, María A. Pajares*1

${ }^{1}$ Instituto de Investigaciones Biomédicas "Alberto Sols" (CSIC-UAM), Arturo Duperier 4, 28029 Madrid (Spain).

${ }^{2}$ Departamento de Bioquímica y Biología Molecular IV, Facultad de Veterinaria, Universidad Complutense de Madrid, 28040 Madrid (Spain).

*Corresponding author: María A. Pajares, Instituto de Investigaciones Biomédicas “Alberto Sols” (CSIC-UAM), Arturo Duperier 4, 28029 Madrid, Spain. Phone number: 34-915854414, FAX number: 34-91-5854401, e-mail:mapajares@iib.uam.es

Evolution of methionine adenosyltransferase 


\section{SUMMARY}

Structural and phylogenetic relationships among Bacteria and Eukaryota were analyzed by examining 292 methionine adenosyltransferase (MAT) amino acid sequences with respect to the crystal structure of this enzyme established for Escherichia coli and rat liver. Approximately $30 \%$ of MAT residues were found to be identical in all species. Five highly conserved amino acid sequence blocks did not vary in the MAT family. We detected specific structural features that correlated with sequence signatures for several clades, allowing taxonomical identification by sequence analysis. In addition, the number of amino acid residues in the loop connecting $\beta$ strands A2 and A3 served to clearly distinguish sequences between eukaryotes and eubacteria. The molecular phylogeny of MAT genes in eukaryotes can be explained in terms of functional diversification coupled to gene duplication or alternative splicing and adaptation through strong structural constraints. Sequence analyses and intron/exon junction positions among nematodes, arthropods and vertebrates support the traditional Coelomata hypothesis. In vertebrates, the liver MAT I isoenzyme has gradually adapted its sequence towards one providing a more specific liver function. MAT phylogeny also served to cluster the major bacterial groups, demonstrating the superior phylogenetic performance of this ubiquitous, housekeeping gene in reconstructing the evolutionary history of distant relatives.

Keywords: Methionine adenosyltransferase, S-adenosylmethionine, evolution, methionine metabolism. 
S-adenosylmethionine (SAM) is the main methyl donor in the transmethylation of numerous essential cell constituents (DNA, neurotransmitters, phospholipids, and many small molecules) ${ }^{1}$. After decarboxylation, SAM acts as a propylamine group donor in the biosynthesis of some polyamines (spermine and spermidine) ${ }^{2}$. Its importance is reflected by the fact that this molecule participates in as many reactions as ATP. However, contrary to ATP which is produced in a large number of reactions, SAM synthesis occurs in only one reaction catalyzed by methionine adenosyltransferase (MAT, EC 2.5.1.6). MAT is generally a homotetrameric enzyme that uses methionine and ATP in a reaction dependent on the presence of $\mathrm{K}^{+}$and $\mathrm{Mg}^{2+}$ ions to render SAM, pyrophosphate and inorganic phosphate ${ }^{3 ; 4}$.

To date, many structure/function relationship studies have used either the Escherichia coli (c-MAT) or rat liver (rl-MAT) enzyme. These studies have provided a relevant amount of information on key residues of the protein, including cysteines and active-site amino acids $5 ; 6 ; 7 ; 8$; ${ }^{9}$. Since the description of the first MAT gene ${ }^{10}$, a substantial number of genes encoding MATs of different origins have been cloned and characterized $11 ; 12 ; 13 ; 14 ; 15 ; 16$. The data obtained indicate exceptional conservation of the gene sequence among highly divergent species. At the amino acid level, c- and rl-MATs have been estimated to show $52 \%$ identity ${ }^{17}$. The crystal structures of these MATs are the only ones presently available that indicate that conservation also occurs at the structural level, and this is reflected by the essentially identical organization of the domains in the monomer ${ }^{7 ; 18}$.

The development of molecular phylogenetics has been generally based on small-subunit (SSU) and large-subunit (LSU) ribosomal RNA analysis ${ }^{19}$. However, several recent concerns have challenged the validity of rRNA as a unique phylogenetic marker. These concerns are related to biases in base composition, disparities in evolutionary rates among lineages, position- 
dependent substitution patterns, alignment ambiguities among very distant species, etc. Thus, recent efforts have focused on assessing the use of other genes ${ }^{20 ; 21 ; 22 ; 23}$ and large combined protein sequence data sets ${ }^{24 ; 25}$ to reconstruct evolutionary relationships among organisms. It has even been suggested that it will be possible to reconstruct a robust universal phylogeny only if a core of conserved markers, not affected by lateral gene transfer, is identified ${ }^{26}$.

Despite a high degree of sequence and structural conservation in MAT, a large number of representative species for which there are available sequences, and vast knowledge on MAT structure and functionality in highly divergent species, this enzyme has not yet been considered as a possible phylogenetic marker. Although attempts have been made to reconstruct partial phylogenies ${ }^{15 ; 27 ; 28}$ and MAT has been included in studies examining sets of several proteins 29 ; 30; 31 , no universal phylogenetic evaluation has used MAT as the marker. Hence, the aim of the present study was to assess the performance of MAT in phylogenetic reconstructions using the 292 sequences available to date, and to demonstrate its utility in molecular systematic studies. 


\section{Results and Discussion}

\section{MAT sequence identification and characterization}

MAT sequences retrieved by database mining led to the identification of 303 candidate sequences in almost every eukaryote and bacterium, but none in archaea, for which non homologous replacement by a new type of MAT has been recently described ${ }^{32}$. The absence of the MAT gene from the complete genomes of members of the genus Chlamydia and the microsporidia Encephalitozoon cuniculi, both intracellular parasites 33; 34, is remarkable. Sequences for Rickettsia prowazekii and R. typhi, also obligate intracellular parasites, were excluded from the analysis because of the recent detection of stop codons in their MAT gene sequences. This may be interpreted as indicative of a certain degree of genome degeneration ${ }^{35}$. The lack or degeneration of the MAT gene in these species may be explained by functional redundancy (obsolescence) or by the existence of another methyl donating pathway (the host produces the methyl donor to be used by the parasite or another compound replaces SAM).

Giardia lamblia, one ancient eukaryote, showed highly divergent sequence and was thus only included in preliminary studies, in which it appeared as the earliest eukaryote with unique structural features. G. lamblia has a 41 amino acid insertion in the loop that connects helix 4 with $\beta$-strand $\mathrm{A} 4$, the significance of which is unknown .

\section{Sequence conservation vs. 3D structure constraints}

A final alignment of 392 positions was obtained from the 292 MAT amino acid sequences considered. Positional identity was difficult to establish in areas located at the N- and C-terminals and at loops connecting secondary structure elements. These ambiguous positions were therefore excluded, leaving 330 parsimony-informative positions for the final analysis. 
MAT protein alignment revealed the presence of 57 amino acids located in identical positions in $100 \%$ of MATs analyzed and 61 additional residues that were conserved in $90 \%$ of the species studied. This indicates that approximately $30 \%$ of MAT residues are identical in all species. Moreover, 49 amino acid positions were identical in $75-90 \%$ of the species, 45 in $60-75 \%$, and 39 in 50-60\%. Residue conservation was inhomogeneous along the MAT sequence. Further, regions such as the $\mathrm{N}$-terminal, $\mathrm{C}$-terminal and some intermediate regions showed a lower degree of identity, probably due the capacity of these areas to absorb a high variety of substitutions without affecting the overall conformation of the molecule and its function.

Core areas of greatest amino acid conservation were observed along 5 stretches which we denoted blocks I, II, III, IV and V (Figure 1). Block I comprises residues 20-47, including the $\beta$ strand A1 and the $\alpha$-helix 1 , and one of the methionine binding motifs, ${ }^{29} \mathrm{GHPDK}^{33}$ which is preserved in all MAT sequences ${ }^{7}$. Block II is defined by two separate areas bearing residues 114-122 and 132-143, flanking the flexible loop at the active site of the enzyme. This loop has been recently shown to be involved in controlling the catalytic efficiency of the enzyme ${ }^{36}$. Conservation of these two areas could be due to the need to preserve the correct orientation for the loop, or to the fact that it contains the ATP binding motif ${ }^{132}$ GAGDQG ${ }^{137}{ }^{17}$. The consensus sequence for ATP binding sites has been defined as GxGDxG plus a lysine located 16-28 residues upstream ${ }^{37}$. However, MAT seems to show no variation in this sequence, which is always GAGDQG, highlighting the significant role of alanine and glutamine residues in this enzyme's ATP binding motif. Block III comprises residues 177-189 that form part of $\beta$-strand A2, including D180 and K182, two of the amino acids involved in catalysis ${ }^{7 ; 18}$. Block IV is the largest and most conserved including 54 amino acids (246-300). This block contains the central loop connecting the $\mathrm{N}$-terminal and central domains, as well as many residues directly involved 
in substrate and cation binding ${ }^{7 ; 18}$. In addition, this block contains a high-glycine stretch (254281 with 10 Gly), which is fully preserved in all MATs. Finally, block V corresponds to the Cterminal and includes $\alpha$-helix 9 (372-389). Besides these blocks, two further reasonably conserved regions were detected: the first comprises $\beta$-strands B1 and B2 with two conserved motifs, residues 55-59 and 70-75, and residue E58, involved in a saline bond with the central loop ${ }^{7}$; and the second area includes residues 303-337, which form part of $\beta$-strands $C 2, \mathrm{C} 3$, and the end of helix 5. However, it must be emphasized that all the amino acids involved in substrate binding and catalysis occur in the blocks described above, and are fully conserved in the MAT family.

To asses the relationship between evolutionary conservation and surface accessibility, the amino acids were also classified according to the degree of identity among MAT sequences as four categories: fully (100\%), highly (75-99\%), moderately (50-75\%) and poorly conserved $(<50 \%)$ (Figure 2). Buried residues were often observed among the amino acids within the first and second categories (>75\%), whereas exposed amino acids were poorly represented in these categories. This over-representation of buried residues among the most conserved residues could reflect their involvement in catalytic activity, correct folding and the stability of the final structure. The fact that they establish the highest number of interactions among residues in the protein structure and their hydrophobic character, may be indicative of their role in the folding nuclei of the monomeric intermediate, according to the overall folding mechanism that has been established ${ }^{38}$. This area is later involved in the association process that leads to the dimer, the minimum active unit of MAT enzymes ${ }^{2}$.

Surface mapping of the level of evolutionary conservation for each amino acid in the protein structure may help to identify functionally and/or structurally significant regions ${ }^{39}$. For 
this purpose, we used available crystallographic data for rl-MAT I/III and color-coded the surface according to the previously defined residue conservation categories (Figure 3A-D). The conservation pattern shows that the preserved blocks defined above occur in the inner channel, where the active site is located, and in the conserved area exposed at both the entrance and walls of this channel. High conservation among residues at the subunit interface is, therefore, consistent with a role for these amino acids in the structure and function of MAT, as reported for other oligomeric enzymes ${ }^{40}$. The study of individual enzyme families reveals how binding and catalysis are optimized in nature through the inclusion of mutations that improve efficiency in cases in which no new function has been acquired ${ }^{41}$. However, the perfect conservation of active site residues in MATs is an exception to this rule, since it indicates the preservation of the catalytic mechanism during evolution with no modification. This would suggest that the special features of the reaction catalyzed by MAT cannot be easily improved.

It is also of interest that several residues outside the subunit interface are also highly conserved. These residues were found to be mainly located in loops connecting secondary structure elements, thus suggesting a key role in preserving the correct orientation between them. The relevance of preserving this orientation probably reflects their essential contribution to final protein folding.

Sequence alignment using conventional algorithms revealed $60 \%$ identity between eukaryotic and bacterial MATs. The high degree of sequence conservation indicates severe restrictions for the substitution of certain amino acids. Such restrictions may be determined by two factors. First, the location of certain residues in the active site is important because of their role in catalysis. Mutations affecting these or adjacent residues modify their relative orientation leading to considerably reduced enzyme activity ${ }^{7}$. The second factor is that correct orientation 
and positioning of certain secondary structure elements seems to depend strongly on the presence of some amino acids in the connecting loops, thus their substitution may lead to wrongly folded structures with no activity. To date, no such modifications have been identified in any MAT sequence though these loops have not yet been mapped by site-directed mutational analysis. Evolutionary studies could, nevertheless, take advantage of these restrictions in the sequence. Residue changes in areas of low variation that could be functionally absorbed in a certain evolutionary setting, are very unlikely to be repeated or to revert back at a different evolutionary time. Thus, the mutational study of certain areas of the MAT sequence may serve to clarify certain evolutionary relationships due to the low probability of mutational saturation at the protein level.

\section{MAT as a phylogenetic marker}

To establish whether MAT could be a useful tool for reconstructing phylogenetic relationships among clades, we used a final data set of 330 positions for distance and parsimony phylogenetic analyses (Figure 4). A general view of the unrooted MAT tree shows the separate grouping of Eukaryota and Eubacteria with high bootstrap support indicating a common evolutionary origin. Protein structure comparisons showed that sequences belonging to each group can be clearly distinguished according to the number of residues involved in the loop connecting $\beta$-strands A2 and A3. Specifically, this loop was normally 3-4 residues shorter in bacteria, except for Campylobacter jejuni and Deinococcus radiodurans. This difference has structural implications as shown in Figure 5. A longer loop allows the establishment of 18 favorable interactions between $\mathrm{N}$ - and C-terminal domains, including the formation of a salt bridge between D192 and R313. However, in eubacteria, only 2 can be formed among such 
interactions including a salt bridge and hence both domains remain more distant. Close inspection of the structural models constructed from the crystallographic data available, shows higher rigidity for the monomer in the eukaryota, due to contact of $\mathrm{N}$ - and C-terminal domains ${ }^{7 \text {; }}$ 18

Members of the MAT family can be grouped into several clades, mostly corresponding to the main taxonomy arrangements. Analysis of MAT phylogeny allows the identification of consensus sequences and specific structural features for the groups (table 2). Additional specific characteristics for each major taxonomic group are detailed below:

\section{Eukaryotes}

A general view of the eukaryotic MAT phylogeny supports an animal-fungal clade excluding green plants. This is consistent with results obtained using SSU rRNA ${ }^{42}$ and protein reconstructions $43 ; 44 ; 45$.

\subsection{Viridiplantae}

Three to four MAT gene copies were identified in the plants. These show differential expression among tissues and during development $27 ; 28 ; 46 ; 47 ; 48 ; 49$. Our analysis also supports (bootstrap value $>99 \%$ ) previous plant MAT gene classification as two types (types I and II) ${ }^{27}$. This topology suggests ancestral duplication, but the absence of MAT genes in conifers prevents us knowing whether duplication occurred before or after the divergence of Magnoliophyta from Coniferophyta. MAT type I duplications indicate low sequence divergence, thus duplication events are likely to have occurred in a narrow time frame. Consequently, phylogenetic relationships in plants cannot be confidently resolved by sequence similarity analysis. The functional requirements of other MAT isoenzymes may stem from their differential regulation 
and/or from new SAM functions observed in plants (e.g., production of the phytohormone ethylene) ${ }^{50}$.

It is generally accepted that the ancestors of vascular plants were similar to green algae (Chlorophyta). In our phylogenetic analysis, the green alga Chlamydomonas reinhardtii branches earliest from the plant clade with high bootstrap support (100\%). Some genes may be nonfunctional because of extensive divergence (e.g., Cicer arieticum) or loss of the C-terminal conservation shown by the remaining MATs (e.g., Gossypium hirsutum 3). Some of these MAT copies may no longer be needed as in other gene duplications and thus degenerate to pseudogenes.

The MAT genes corresponding to conifers appeared apart from the remaining plants, indicating earlier divergence for this group. The inclusion of one of the two genes isolated from Pinus contorta among the Leuconostocaceae, which were initially considered an outgroup for the plants, deserves special mention ${ }^{28}$. Based on the high degree of confidence for this node, the most parsimonious explanation for this would be a horizontal gene transfer event.

\subsection{Fungi}

In this phylogenetic analysis, fungi clustered as a monophyletic group forming the second branch point from that leading to the animals. This topology is well-supported by other studies 51. Moreover, the MAT tree shows the early separation of the phylum Basidiomycota from Ascomycota, with high bootstrap support (100\%). Only one MAT gene could be identified in most of the fungal species. The exception was Saccharomyces cerevisiae which has recently duplicated its genome ${ }^{52}$ and has two similar copies of the MAT gene.

\subsection{Nematoda}


Interestingly, Caenorhabditis elegans presents 5 MAT genes. One of them, C49F5.1, was separated by an earlier gene duplication explaining its large phylogenetic distance and least similarity to the other $C$. elegans MATs. Genome analysis of the MAT genes yielded the following observations: a) four genes on autosomic chromosome IV, whereas C49F5.1 appears X-linked; b) this last gene presents 4 exons compared to the others showing 6; and c) three MAT genes were identified in C. briggsae, one of which showed similar characteristics to C49F5.1. Thus, MAT genes in nematodes probably arose from one single copy that underwent duplication, leading to genes on chromosomes X and IV. Further duplications led to the four copies identified on chromosome IV. Divergences among the five gene sequences could be attributed to a higher mutational rate of the genes on chromosome $\mathrm{X}$, or to the fact that the duplications in chromosome IV have recently taken place. Just as MAT type II in plants, C49F5.1 seems to be the result of a duplication event required for different regulation or new functions of the final reaction product, SAM.

\subsection{Arthropoda}

Arthropods differed from the other organisms containing specialized tissues in that only one MAT gene was identified. However, genomic analysis revealed the possibility that this gene may suffer alternative splicing of exon 4 which codifies the flexible loop. Differences among both exons occur in the highly variable region within the loop, whereas conserved regions remain unchanged. These results suggest a role for the flexible loop in the regulation of arthropod MAT that eliminates the need for duplication and tissue specific distribution for each gene, i.e., the most common mechanism seen in MAT from higher organisms.

\subsection{Vertebrata}


There are three isoforms of MAT in mammalian tissues that are encoded by two genes. MAT I and MAT III are tetrameric and dimeric forms, respectively, of the same gene product (MAT1A), which is mainly expressed in adult liver ${ }^{53}$. The ubiquitously expressed MAT II isoform is a heterotetramer $\left(\alpha_{2} \beta_{2}\right)$, whose catalytic subunit $(\alpha)$ is encoded by a different gene $(\mathrm{MAT} 2 \mathrm{~A})^{2}$. The protein isoforms also differ in their regulation and catalytic properties (affinities for methionine) ${ }^{2 ;} 53$. In every vertebrate reported here, both genes presented around $85 \%$ identity, differences always corresponding to the same regions. The MAT tree clearly separates both gene types and locates the duplication event after the divergence from Urochordata, but before the divergence from Teleostomi. This is not surprising, if we consider that many early chordate gene families were formed or expanded by large-scale DNA duplications ${ }^{54}$. Interestingly, liver ontogeny in vertebrates is concomitant with this MAT gene duplication suggesting that tissue-specialized enzyme forms were required for adaptation to the functions of this new organ. MAT I/III has been suggested as a marker for liver development, due to the differential expression of its isoforms in fetal and adult tissue ${ }^{55}$.

A further significant issue is identifying specific isozyme residues to which to ascribe functional roles for these conserved amino acids, such as has been done for other homotetrameric enzymes ${ }^{56 ; 57}$. This identification uses information from the sequence alignment of vertebrate MAT isozymes and is based on the criterion that the residue is conserved in orthologs and is distinct among paralogs. Surprisingly, not all the residues considered liver-specific occurred in all our vertebrate clades suggesting that the specialization of the liver MAT gene took place gradually. Thus, cysteine 121 is found at the flexible loop of the liver enzyme and, being the target for regulation by nitrosylation of the protein ${ }^{58}$, it mediates the response to oxidative stress. This residue is present in mammalian liver MAT, but not in that of Gallus gallus. By comparing 
MAT I/III and MAT II among mammals, birds, amphibian and fishes we were able to identify further analogous positions (Table 2). Along with functional studies, the characterization of new MAT sequences from species intermediate between mammals and ancient chordates, may clarify the evolutionary adaptation related to functional diversification of the MAT liver gene.

Finally, sequence comparison and the evolution of intron/exon junction positions among bilateria (data not shown) suggest the presence of a common ancestor for arthropods and vertebrates that branched from nematodes. This observation is in line with previous studies based on $18 \mathrm{~S}$ rRNA that assign nematodes and arthropods to a common clade (Ecdysozoa) separate from vertebrates ${ }^{59}$. Conversely, our results are more in agreement with a recent study based on more than 100 proteins that supports the traditional Coelomata hypothesis ${ }^{31}$ grouping arthropods with vertebrates apart from nematodes. This reinforces the idea that MAT is a better marker than rRNA for resolving the branching order of the main animal lineages.

\section{Bacteria}

Five clades of bacteria were clearly distinguished in our molecular phylogeny of MAT based on the presence of a single gene. These clades are: Proteobacteria, Cyanobacteria, CFB group, Actinobacteria and Firmicutes. Single representative species of several phyla appear as independent branches of the tree: Aquificae (A. aeolicum), Thermotogae (T. maritima), Deinococcus-Thermus (D. radiodurans), Chloroflexi (C. aurianticus), Planctomycetes (G. obscuriglobis), Fibrobacteres (F. succinogenes), Spirochaetes (B. burgdorferi) and Fusobacteria (F. nucleatum). The anaerobic detoxifying bacterium Dehalococcoides ethenogenes also appeared as an independent branch confirming its position in a unique phylogenetic group as described by the use of $16 \mathrm{~S}_{\mathrm{rRNA}}{ }^{60}$. There is little resolution in the MAT tree reflecting the 
position of these phyla, and hence no consistent phylogenetic relationships with other groups could be clearly established.

\subsection{Proteobacteria}

Proteobacteria have been previously divided into five phylogenetically distinct groups $(\alpha$, $\beta, \gamma, \delta$ and $\varepsilon)^{19}$. In the present phylogenetic analysis, proteobacterial MAT sequences did not consistently form a clade. However, the $\alpha-\beta-, \gamma$ - and $\delta$ - groups were found to cluster individually.

\subsection{1 $\alpha$-Proteobacteria}

All the $\alpha$-proteobacteria MAT considered grouped with near $100 \%$ confidence but branched apart from the rest of proteobacteria because of the extension of several loops only detected in this class (Table 2). The phylogenetic tree presents an early branching point bearing several members of the order Rickettsiales, indicating the early separation of this order from the remaining $\alpha$-proteobacteria. The order Rhizobiales was also clearly separated from the rest of the $\alpha$-proteobacteria. The most distinctive feature of species of this order was the presence of a 7 amino acid insertion between helix 1 and $\beta$-strand B1. Other characteristics typical of rhizobial MATs were: a) a flexible loop at least 2 residues longer than in the other $\alpha$-proteobacteria; and b) an insertion between $\beta$-strand B2 and helix 2. The exception to this rule was shown by Methylobacterium extorquens with none of these peculiarities, indicating the very early separation of this family from the remaining rhizobiales.

\subsection{2 $\beta$-Proteobacteria}

Based on this phylogenetic tree, MAT sequences from $\beta$ - and $\gamma$-proteobacteria show a common origin, whereas relationships with the rest of the proteobacteria remain obscure, given the $<50 \%$ bootstrap value for the nodes separating these bacterial classes. Differences between 
both groups rely, for example, on the presence of two extra residues in the flexible loop of the $\beta$ proteobacteria. Of interest is the presence of Acidithiobacillus ferrooxidans among the $\beta$ proteobacteria, despite its taxonomical classification as belonging to the order Chromatiales of the $\gamma$-proteobacteria. However, the high statistical support for this branch in our analysis and the absence of the typical characteristics of a $\gamma$-proteobacteria suggest its reclassification within $\beta$ proteobacteria.

\subsection{3 $\gamma$-Proteobacteria}

Most of the $\gamma$-proteobacteria clustered in the tree, showing relationships among the different orders in this class. All the members of the Pasteurellales included in the tree grouped together, as occurred for the Enterobacteria. MAT sequences in members of the order Legionellales slightly diverged from those of the remaining $\gamma$-proteobacteria. This leads to the early branching of Legionellales from the $\gamma$-proteobacteria group.

\subsection{Cyanobacteria}

Based on MAT phylogeny, the Cyanobacteria appeared as an independent taxonomic group that was well differentiated by specific insertions (Table 2). Two of the four members of subsection I included in this study (Prochlorococcus marinus and Synechococcus sp.) carried a 7-residue insertion between helix 4 and $\beta$-strand A4. In contrast, remaining cyanobacteria only have 6 amino acids at this position. In addition, these two species have an extra residue inserted in the loop between helix 2 and $\beta$-strand B3. This could explain why $P$. marinus and Synechococcus sp. occur apart from the other members of subsections I, III and IV.

\subsection{CFB group}

The CFB group was included in a homogeneous cluster due to the specific characteristics of this phylum (Table 2). It should be highlighted that phylogenetic reconstruction places 
Chlorobium tepidum on an early branch from the CFB group, although it presents distinctive features such as a 4-residue insertion in the flexible loop, and the absence of insertions in the loop between helix 4 and $\beta$-strand A4. This position in the tree indicates the existence of a common ancestor for both phyla and their subsequent divergence. The class Sphingobacteria reflects this group's peculiarities: the loop between $\beta$-strand A3 and helix 4 does not include the 10-12 residue insertion detected in members of the class Bacteroidetes. Moreover, in Chlorobium this insertion bears 7 residues.

\subsection{Actinobacteria}

Members of the group Actinomycetales clustered perfectly together, with the order Bifidobacteriales remaining apart. The following modifications to the general characteristics were, nevertheless, observed: a) longer insertions in the flexible loop in B. longum and $S$. fradiae; b) Mycobacterium avium, M. leprae and M. tuberculosis have a 3-residue insertion in the loop connecting $\beta$-strand B2 and helix 2 ; c) the insertion in the loop between helix 4 and $\beta$ strand A4 is absent in Thermobifida and differs in length between Streptomyces (4 residues) and Mycobacterium, Corynebacterium and Bifidobacterium (3-2 residues).

\subsection{Firmicutes}

2.5.1 Bacilli/Clostridia. All Clostridia and Bacilli clustered together but separately defined each class. Moreover, within Bacilli, the orders Bacillales and Lactobacillales were also separately arranged. The topology observed for the family Leuconostaceae is an exception, which can be explained by the early divergence from a common ancestor giving rise to a branch for Leuconostocaceae and a later divergence rendering the Bacilli and Clostridia families. Alternatively, the MAT sequence in Leuconostocaceae diverged rapidly.

\subsubsection{Mollicutes}


This class clustered apart from the other Firmicutes (Clostridia, Bacilli). This might be explained by the presence of a 7-amino acid deletion in the loop connecting helix 2 and $\beta$-strand B3. Mycoplasma pneumoniae and M. genitalium clustered apart from M. pulmoni and U. urealyticum. These last species presented a 2-amino acid insertion in the loop between helices 8 and 9. In contrast, the separation of $M$. pneumoniae and $M$. genitalium may be attributed to differential structural features: a) a flexible loop two amino acids shorter; b) a residue inserted in the loop between helix 4 and $\beta$-strand A4; c) another residue inserted in the loop connecting $\beta$ strand $\mathrm{C} 3$ and helix 6; and d) the insertion of a residue in the loop between helices 7 and 8 . In addition, the positioning of Mycoplasma among this group is congruent with results derived from protein fusion trees, in contrast to rRNA trees ${ }^{26}$. This finding is yet another confirmation of the idea that protein sequences more accurately reflect the phylogenetic position of Mycoplasma ${ }^{61}$.

\section{Conclusions}

The present study is a first attempt at using the housekeeping MAT gene as a marker in eukarya and bacterial systematics, as an alternative to rRNA and other protein reference markers. This work is the result of integrating data yielded by intensive data mining, robust aligning of MAT sequences and structural-functional analyses. Through the detection of fully conserved regions in the MAT protein of all species, regions varying even among close relatives and characteristic structural features missing from certain taxonomic groups, this new tool enabled us to resolve phylogenetic relationships between close and distant relatives with high bootstrap support. 


\section{Materials and Methods}

\section{MAT sequences}

MAT amino acid sequences were deduced from DNA sequence data available from complete or nearly complete publicly available genomes by conducting a TBLASTN search using rl-MAT as probe. For further references to amino acid positions rl-MAT is used as the consensus sequence. Candidate sequences were identified as MAT when they met the following criteria: a) a length of 370-414 amino acids; b) an N-terminal sequence containing the motif ${ }^{21}$ FTSESVxEGHPDK ${ }^{33}$; and c) a C-terminal including the motif ${ }^{379} \mathrm{GHFGxxxxWE}^{389}$. MAT sequences were obtained from GenBank, The Institute for Genomic Research (TIGR), DOE Joint Genome Institute, The Sanger Institute, University of Oklahoma, Baylor College of Medicine, Genome Sequencing Center at Washington University Medical School, Columbia Genome Center, Whitehead/MIT Genome Center, Genoscope, DNA Data Bank of Japan, Université Catholique de Louvain and European Bioinformatics Institute. A complete list of the sequences and their sources is provided in table 1.

\section{Alignment and sequence analysis}

Sequences were aligned using the program BIOEDIT ${ }^{62}$. The alignment was manually refined to correct for large inserts and ambiguities. Final alignments contained 292 sequences 370-414 amino acids long from which 392 positions were selected for phylogenetic reconstruction. A total number of 330 informative positions were taken into account. Identity percentages were calculated using MULTALIN ${ }^{63}$. For additional information visit our database home page at http://www.iib.uam.es/MAT. 


\section{Evolutionary analysis}

MAT's evolutionary tree was established from the 292 sequence alignment of the 303 sequences available using neighbor-joining and parsimony methods. MAT sequences for the following microorganisms were discarded in the final analysis due to ambiguity: Amoeba proteus, Campylobacter jejuni, Escherichia coli II, Giardia lamblia, Mesorhizobium loti II, Nostoc sp. PCC 7120. II, Rickettsia prowazekii, Rickettsia typhi, Treponema pallidum, Leptospira interrogans and Tropherma whipplei. Ambiguity may arise when there is a loss in the phylogenetic signal, obsolescence, insufficient taxon sampling or high sequence divergence. The phylogenetic analysis was performed using the PHYLIP package v.3.5 ${ }^{64}$. Distances were calculated using Dayhoff in PROTDIST followed by the use of NEIGHBOR or PROTPARS for tree reconstruction. Phylogenetic trees were drawn using the MEGA2 ${ }^{65}$ and ATV programs ${ }^{66}$. Statistical support for the groups in the tree was evaluated by bootstrap analysis of 500 iterations using SEQBOOT.

\section{Amino acid solvent accessibility}

Coordinates for rl-MAT crystal structure ${ }^{7}$ were used to calculate the solvent accessibility (SA) of each individual residue in the dimeric structure using the SwissPdbViewer

67. Amino acids with a SA $<10 \%$ are regarded as buried, whereas residues with $\mathrm{SA}>10 \%$ are considered exposed ${ }^{68}$. 


\section{Footnotes}

\section{Acknowledgments}

This work has been supported by grants of Fondo de Investigación Sanitaria of the Instituto de Salud Carlos III (01/1077 and RCMN C03/08) and MCYT (BMC-2002-00243) (to M.A.P.), and MCYT (PM99-0049-C02-01) (to J.M.B.).

Abbreviations: MAT, ATP:L-methionine adenosyltransferase; c-MAT, E. coli methionine adenosyltransferase; rl-MAT, rat liver methionine adenosyltransferase; SAM, Sadenosylmethionine; L-cisAMB, L-2-amino-4-methoxy-cis-but-3-enoic acid. 


\section{References}

1. Cantoni, G. L. (1975). Biological methylation: selected aspects. Annu Rev Biochem 44, 435-51.

2. Mato, J. M., Alvarez, L., Ortiz, P. \& Pajares, M. A. (1997). S-adenosylmethionine synthesis: molecular mechanisms and clinical implications. Pharmacol Ther 73, 265-80.

3. Cantoni, G. L. (1953). S-adenosylmethionine: a new intermediate formed enzymatically from L-methionine and adenosinetriphosphate. J Biol Chem 204, 403-416.

4. McQueney, M. S. \& Markham, G. D. (1995). Investigation of monovalent cation activation of S-adenosylmethionine synthetase using mutagenesis and uranyl inhibition. $J$ Biol Chem 270, 18277-84.

5. Mingorance, J., Alvarez, L., Sanchez-Gongora, E., Mato, J. M. \& Pajares, M. A. (1996). Site-directed mutagenesis of rat liver S-adenosylmethionine synthetase. Identification of a cysteine residue critical for the oligomeric state. Biochem J 315 ( Pt 3), 761-6.

6. Taylor, J. C. \& Markham, G. D. (1999). The bifunctional active site of sadenosylmethionine synthetase. Roles of the active site aspartates. J Biol Chem 274, 32909-14.

7. Gonzalez, B., Pajares, M. A., Hermoso, J. A., Alvarez, L., Garrido, F., Sufrin, J. R. \& Sanz-Aparicio, J. (2000). The crystal structure of tetrameric methionine adenosyltransferase from rat liver reveals the methionine-binding site. J Mol Biol 300, 363-75.

8. Taylor, J. C. \& Markham, G. D. (2000). The bifunctional active site of Sadenosylmethionine synthetase. Roles of the basic residues. J Biol Chem 275, 4060-5.

9. Sanchez-Perez, G. F., Gasset, M., Calvete, J. J. \& Pajares, M. A. (2003). Role of an Intrasubunit Disulfide in the Association State of the Cytosolic Homo-oligomer Methionine Adenosyltransferase. J Biol Chem 278, 7285-7293.

10. Markham, G. D., DeParasis, J. \& Gatmaitan, J. (1984). The sequence of metK, the structural gene for S-adenosylmethionine synthetase in Escherichia coli. J Biol Chem 259, $14505-7$.

11. Thomas, D. \& Surdin-Kerjan, Y. (1987). SAM1, the structural gene for one of the Sadenosylmethionine synthetases in Saccharomyces cerevisiae. Sequence and expression. J Biol Chem 262, 16704-9.

12. Larsson, J. \& Rasmuson-Lestander, A. (1994). Molecular cloning of the Sadenosylmethionine synthetase gene in Drosophila melanogaster. FEBS Lett 342, 329-33.

13. Mautino, M. R., Barra, J. L. \& Rosa, A. L. (1996). eth-1, the Neurospora crassa locus encoding S-adenosylmethionine synthetase: molecular cloning, sequence analysis and in vivo overexpression. Genetics 142, 789-800.

14. Yocum, R. R., Perkins, J. B., Howitt, C. L. \& Pero, J. (1996). Cloning and characterization of the metE gene encoding S-adenosylmethionine synthetase from Bacillus subtilis. J Bacteriol 178, 4604-10.

15. Chiang, P. K., Chamberlin, M. E., Nicholson, D., Soubes, S., Su, X., Subramanian, G., Lanar, D. E., Prigge, S. T., Scovill, J. P., Miller, L. H. \& Chou, J. Y. (1999). Molecular characterization of Plasmodium falciparum S-adenosylmethionine synthetase. Biochem J 344 Pt 2, 571-6.

16. Reguera, R. M., Balana-Fouce, R., Perez-Pertejo, Y., Fernandez, F. J., Garcia-Estrada, C., Cubria, J. C., Ordonez, C. \& Ordonez, D. (2002). Cloning expression and 
characterization of methionine adenosyltransferase in Leishmania infantum promastigotes. J Biol Chem 277, 3158-67.

17. Horikawa, S., Ishikawa, M., Ozasa, H. \& Tsukada, K. (1989). Isolation of a cDNA encoding the rat liver S-adenosylmethionine synthetase. Eur J Biochem 184, 497-501.

18. Takusagawa, F., Kamitori, S. \& Markham, G. D. (1996). Structure and function of Sadenosylmethionine synthetase: crystal structures of S-adenosylmethionine synthetase with ADP, BrADP, and PPi at 28 angstroms resolution. Biochemistry 35, 2586-96.

19. Woese, C. R. (1987). Bacterial evolution. Microbiol Rev 51, 221-71.

20. Gupta, R. S. \& Golding, G. B. (1993). Evolution of HSP70 gene and its implications regarding relationships between archaebacteria, eubacteria, and eukaryotes. J Mol Evol 37, 573-82.

21. Gupta, R. S., Aitken, K., Falah, M. \& Singh, B. (1994). Cloning of Giardia lamblia heat shock protein HSP70 homologs: implications regarding origin of eukaryotic cells and of endoplasmic reticulum. Proc Natl Acad Sci U S A 91, 2895-9.

22. Eisen, J. A. (1995). The RecA protein as a model molecule for molecular systematic studies of bacteria: comparison of trees of RecAs and 16S rRNAs from the same species. J Mol Evol 41, 1105-23.

23. Baldauf, S. L., Palmer, J. D. \& Doolittle, W. F. (1996). The root of the universal tree and the origin of eukaryotes based on elongation factor phylogeny. Proc Natl Acad Sci U S A 93, 7749-54.

24. Brown, J. R., Douady, C. J., Italia, M. J., Marshall, W. E. \& Stanhope, M. J. (2001). Universal trees based on large combined protein sequence data sets. Nat Genet 28, 281-5.

25. Baldauf, S. L., Roger, A. J., Wenk-Siefert, I. \& Doolittle, W. F. (2000). A kingdom-level phylogeny of eukaryotes based on combined protein data. Science 290, 972-7.

26. Brochier, C., Bapteste, E., Moreira, D. \& Philippe, H. (2002). Eubacterial phylogeny based on translational apparatus proteins. Trends Genet 18, 1-5.

27. Schroder, G., Eichel, J., Breinig, S. \& Schroder, J. (1997). Three differentially expressed S-adenosylmethionine synthetases from Catharanthus roseus: molecular and functional characterization. Plant Mol Biol 33, 211-22.

28. Lindroth, A. M., Saarikoski, P., Flygh, G., Clapham, D., Gronroos, R., Thelander, M., Ronne, H. \& von Arnold, S. (2001). Two S-adenosylmethionine synthetase-encoding genes differentially expressed during adventitious root development in Pinus contorta. Plant Mol Biol 46, 335-46.

29. Feng, D. F., Cho, G. \& Doolittle, R. F. (1997). Determining divergence times with a protein clock: update and reevaluation. Proc Natl Acad Sci U S A 94, 13028-33.

30. Mushegian, A. R., Garey, J. R., Martin, J. \& Liu, L. X. (1998). Large-scale taxonomic profiling of eukaryotic model organisms: a comparison of orthologous proteins encoded by the human, fly, nematode, and yeast genomes. Genome Res 8, 590-8.

31. Blair, J. E., Ikeo, K., Gojobori, T. \& Hedges, S. B. (2002). The evolutionary position of nematodes. BMC Evol Biol 2, 7.

32. Graham, D. E., Bock, C. L., Schalk-Hihi, C., Lu, Z. J. \& Markham, G. D. (2000). Identification of a highly diverged class of S-adenosylmethionine synthetases in the archaea. J Biol Chem 275, 4055-9.

33. Wicher, V., Baughn, R. E., Fuentealba, C., Shadduck, J. A., Abbruscato, F. \& Wicher, K. (1991). Enteric infection with an obligate intracellular parasite, Encephalitozoon cuniculi, in an experimental model. Infect Immun 59, 2225-31. 
34. Zomorodipour, A. \& Andersson, S. G. (1999). Obligate intracellular parasites: Rickettsia prowazekii and Chlamydia trachomatis. FEBS Lett 452, 11-5.

35. Andersson, J. O. \& Andersson, S. G. (1999). Genome degradation is an ongoing process in Rickettsia. Mol Biol Evol 16, 1178-91.

36. Taylor, J. C., Takusagawa, F. \& Markham, G. D. (2002). The active site loop of Sadenosylmethionine synthetase modulates catalytic efficiency. Biochemistry 41, 9358-69.

37. Wierenga, R. K., Terpstra, P. \& Hol, W. G. (1986). Prediction of the occurrence of the ADP-binding beta alpha beta-fold in proteins, using an amino acid sequence fingerprint. $J$ Mol Biol 187, 101-7.

38. Gasset, M., Alfonso, C., Neira, J. L., Rivas, G. \& Pajares, M. A. (2002). Equilibrium unfolding studies of the rat liver methionine adenosyltransferase III, a dimeric enzyme with intersubunit active sites. Biochem J 361, 307-15.

39. Glaser, F., Pupko, T., Paz, I., Bell, R. E., Bechor-Shental, D., Martz, E. \& Ben-Tal, N. (2003). ConSurf: identification of functional regions in proteins by surface-mapping of phylogenetic information. Bioinformatics 19, 163-4.

40. Notaro, R., Afolayan, A. \& Luzzatto, L. (2000). Human mutations in glucose 6phosphate dehydrogenase reflect evolutionary history. Faseb $J$ 14, 485-94.

41. Todd, A. E., Orengo, C. A. \& Thornton, J. M. (2002). Plasticity of enzyme active sites. Trends Biochem Sci 27, 419-26.

42. Van de Peer, Y., Baldauf, S. L., Doolittle, W. F. \& Meyer, A. (2000). An updated and comprehensive rRNA phylogeny of (crown) eukaryotes based on rate-calibrated evolutionary distances. J Mol Evol 51, 565-76.

43. Stechmann, A. \& Cavalier-Smith, T. (2002). Rooting the eukaryote tree by using a derived gene fusion. Science 297, 89-91.

44. Roger, A. J., Sandblom, O., Doolittle, W. F. \& Philippe, H. (1999). An evaluation of elongation factor 1 alpha as a phylogenetic marker for eukaryotes. Mol Biol Evol 16, 21833.

45. Baldauf, S. L. (1999). A Search for the Origins of Animals and Fungi: Comparing and Combining Molecular Data. Am Nat 154, S178-S188.

46. Espartero, J., Pintor-Toro, J. A. \& Pardo, J. M. (1994). Differential accumulation of Sadenosylmethionine synthetase transcripts in response to salt stress. Plant Mol Biol 25, 217-27.

47. Vander Mijnsbrugge, K., Van Montagu, M., Inze, D. \& Boerjan, W. (1996). Tissuespecific expression conferred by the S-adenosyl-L-methionine synthetase promoter of Arabidopsis thaliana in transgenic poplar. Plant Cell Physiol 37, 1108-15.

48. Lee, J. H., Chae, H. S., Hwang, B., Hahn, K. W., Kang, B. G. \& Kim, W. T. (1997). Structure and expression of two cDNAs encoding S-adenosyl-L-methionine synthetase of rice (Oryza sativa L.). Biochim Biophys Acta 1354, 13-8.

49. Gomez-Gomez, L. \& Carrasco, P. (1998). Differential expression of the S-adenosyl-Lmethionine synthase genes during pea development. Plant Physiol 117, 397-405.

50. Kende, H. (1993). Ethylene biosynthesis. Annu Rev Plant Physiol Plant Mol Biol 44, 283-307.

51. Baldauf, S. L. \& Palmer, J. D. (1993). Animals and fungi are each other's closest relatives: congruent evidence from multiple proteins. Proc Natl Acad Sci U S A 90, 11558-62. 
52. Wolfe, K. H. \& Shields, D. C. (1997). Molecular evidence for an ancient duplication of the entire yeast genome. Nature 387, 708-13.

53. Lu, S. C., Gukovsky, I., Lugea, A., Reyes, C. N., Huang, Z. Z., Chen, L., Mato, J. M., Bottiglieri, T. \& Pandol, S. J. (2003). Role of S-adenosylmethionine in two experimental models of pancreatitis. Faseb $J 17,56-8$.

54. McLysaght, A., Hokamp, K. \& Wolfe, K. H. (2002). Extensive genomic duplication during early chordate evolution. Nat Genet 31, 200-4.

55. Gil, B., Casado, M., Pajares, M. A., Bosca, L., Mato, J. M., Martin-Sanz, P. \& Alvarez, L. (1996). Differential expression pattern of S-adenosylmethionine synthetase isoenzymes during rat liver development. Hepatology 24, 876-81.

56. Chang, S. H. \& Kemp, R. G. (2002). Role of Ser530, Arg292, and His662 in the allosteric behavior of rabbit muscle phosphofructokinase. Biochem Biophys Res Commun 290, 670-5.

57. Pezza, J. A., Choi, K. H., Berardini, T. Z., Beernink, P. T., Allen, K. N. \& Tolan, D. R. (2003). Spatial Clustering of Isozyme-specific Residues Reveals Unlikely Determinants of Isozyme Specificity in Fructose-1,6-bisphosphate Aldolase. J Biol Chem 278, 17307 13.

58. Avila, M. A., Mingorance, J., Martinez-Chantar, M. L., Casado, M., Martin-Sanz, P., Bosca, L. \& Mato, J. M. (1997). Regulation of rat liver S-adenosylmethionine synthetase during septic shock: role of nitric oxide. Hepatology 25, 391-6.

59. Aguinaldo, A. M., Turbeville, J. M., Linford, L. S., Rivera, M. C., Garey, J. R., Raff, R. A. \& Lake, J. A. (1997). Evidence for a clade of nematodes, arthropods and other moulting animals. Nature 387, 489-93.

60. Hendrickson, E. R., Payne, J. A., Young, R. M., Starr, M. G., Perry, M. P., Fahnestock, S., Ellis, D. E. \& Ebersole, R. C. (2002). Molecular analysis of Dehalococcoides 16S ribosomal DNA from chloroethene-contaminated sites throughout North America and Europe. Appl Environ Microbiol 68, 485-95.

61. Kamla, V., Henrich, B. \& Hadding, U. (1996). Phylogeny based on elongation factor Tu reflects the phenotypic features of mycoplasmas better than that based on 16S rRNA. Gene 171, 83-7.

62. Hall, T. A. (1999). BioEdit: a user-friendly biological alignment editor and analysis program for Windows 95/98/NT. Nucl Acids Symp Sex 41, 95-98.

63. Corpet, F. (1988). Multiple sequence alignment with hierarchical clustering. Nucleic Acids Res 16, 10881-90.

64. Felsenstein, J. (1993). PHYLIP (phylogeny inference package) version 3.5c. University of Washington, Seattle.

65. Kumar, S., Tamura, K., Jakobsen, I. B. \& Nei, M. (2001). MEGA2: molecular evolutionary genetics analysis software. Bioinformatics 17, 1244-5.

66. Zmasek, C. M. \& Eddy, S. R. (2001). ATV: display and manipulation of annotated phylogenetic trees. Bioinformatics 17, 383-4.

67. Guex, N. \& Peitsch, M. C. (1997). SWISS-MODEL and the Swiss-PdbViewer: an environment for comparative protein modeling. Electrophoresis 18, 2714-23.

68. Goldman, N., Thorne, J. L. \& Jones, D. T. (1998). Assessing the impact of secondary structure and solvent accessibility on protein evolution. Genetics 149, 445-58. 


\section{Figure legends}

Figure 1. Evolution of the MAT protein. This diagram shows the analysis of 292 different MAT sequences. Rat liver MAT (lower row) was used as the reference sequence. The lettering for rat liver MAT indicates amino acid solvent accessibility (SA): blue, buried (SA<10\%); black, exposed $(\mathrm{SA}>10 \%)$. Immediately above the rat liver MAT sequence, we show the identity consensus sequence for the 292 sequences analyzed. At each position, the most frequent amino acid is coded according to its frequency (blue: 100\%; green: 90-99\%; red: 75-90\%; yellow: 6075\%). The conserved blocks described in the text are boxed and identified with Roman numerals. The locations of $\alpha$-helices and $\beta$-strands as found in the rat liver MAT 3D structure are provided above the identity consensus sequence. The structure of the first 16 residues (lowercase) in rat liver MAT is not known.

Figure 2. Conservation and 3-dimensional structure. Each bar represents the distribution of MAT amino acids among the different conservation categories for exposed, total, and buried residues, respectively.

Figure 3. Diagram showing MAT sequence conservation in 3-dimensional structure. The conservation pattern is color-coded on the molecular surface of rat liver MAT: dark violet indicates maximal conservation (100-90\% identity), white indicates an average conservation level (90-50\%) and dark turquoise indicates maximal variability (<50\% identity). (A) Dimer viewed from the active site entrance. (B) Side view of the dimer after a $90^{\circ}$ rotation to the right showing the exposed surface. (C) A view of the monomer from the monomer-monomer interface. (D) Side view of the monomer after a $180^{\circ}$ rotation showing the exposed surface. 
Figure 4. Phylogenetic tree derived from MAT sequences. Numbers on the branches show the percentage occurrence of nodes in 500 bootstrap replicates in the neighbor-joining and maximum parsimony analyses. Bootstrap values are indicated only when greater than $70 \%$. For simplicity, the branches within each major group were collapsed.

Figure 5. Differences between rat liver MAT (A) (PDB ID code: 1QM4) and E. coli MAT (B) (PDB ID code: 1fug) for the atomic interactions between loops connecting A2 and A3 strands in the N-terminal domain (yellow ribbons and red backbone and side chains) and loops connecting helix 5, C2 and C3 strands in the C-terminal domain (blue ribbons and cyan backbone and side chains). 


\section{Tables}

Table 1. List of MAT sequences used in the present analysis.

\section{Specie}

Acanthamoeba castellanii

Acidithiobacillus ferroxidans

Acinetobacter ADP-1

Actinidia chinensis 1

Actinidia chinensis 2

Actinobacillus actinomycetemcomitans

Agrobacterium tumefaciens

Ajellomyces capsulatus

Anaplasma phagocytophila

Anopheles gambiae

Aquifex aeolicus

Arabidopsis thaliana 1

Arabidopsis thaliana 2

Arabidopsis thaliana 3

Arabidopsis thaliana 4

Ascaris lumbricoides

Ascobolus immersus

Aspergillus fumigatus

Aspergillus nidulans

Azotobacter vinelandii

Bacillus anthracis

Bacillus cereus 1

Bacillus cereus 2

Bacillus halodurans

Bacillus stearothermophilus

Bacillus subtilis

Bacteroides forsythus

Bacteroides fragilis

Bacteroides thetaiotaomicron

Bifidobacterium longum

Bombyx mori

Bordetella avium

Bordetella bronchiseptica

Bordetella pertussis

Borrelia burgdorferi

Botrytis cinerea

Bradyrhizobium japonicum

Brassica juncea 1

Brassica juncea 2

Brassica juncea 3
Accesion $\mathbf{N}^{\mathbf{0}}$ /

Genome center

$\underline{\mathbf{9 2 7 4 8 7}}$

TIGR

Genoscope

726030

726028

Univ. Oklahoma

17934278

Univ. Washington

TIGR

$\underline{21291484}$

7387875

15217781

$\underline{15234354}$

$\underline{15229033}$

$\underline{15228048}$

EBI Parasites

836960

TIGR

Whitehead/MIT

23102724

$\underline{21402812}$

TIGR

29898393

20138752

Univ. Oklahoma

$\mathbf{7 4 3 4 0 0 8}$

TIGR

Sanger Institute

29340533

$\underline{23327085}$

EST NCBI

Sanger Institute

Sanger Institute

Sanger Institute

$\mathbf{7 4 3 4 0 0 4}$

Genoscope

$\underline{27354222}$

$\underline{10443981}$

$\underline{14600070}$

$\underline{14600072}$ 
Brucella suis

Buchnera aphidicola

Burkholderia cepacia

Burkholderia fungorum

Burkholderia mallei

Burkholderia pseudomallei

Caenorhabditis briggsae 1

Caenorhabditis briggsae 2

Caenorhabditis briggsae 3

Caenorhabditis elegans 1

Caenorhabditis elegans 2

Caenorhabditis elegans 3

Caenorhabditis elegans 4

Caenorhabditis elegans 5

Camellia sinensis

Campylobacter jejuni

Candida albicans

Carboxydothermus hydrogenoformans

Carica papaya

Catharanthus roseus 1

Catharanthus roseus 2

Catharanthus roseus 3

Caulobacter crescentus

Chlamydomonas reinhardtii

Chlorobium tepidum

Chloroflexus aurantiacus

Cicer arietinum

Ciona intestinalis

Clavibacter michiganensis

Clostridium acetobutylicum

Clostridium botulinum

Clostridium perfringens

Clostridium tetani

Coccidioides posadasii

Colwellia psychroerythraea

Corynebacterium diphtheriae

Corynebacterium efficiens

Corynebacterium glutamicum

Coxiella burnetii

Cryptococcus neoformans

Cryptosporidium parvum

Cytophaga hutchinsonii

Danio rerio 1

Danio rerio 2

Dechloromonas aromatica

Dehalococcoides ethenogenes

Deinococcus radiodurans

\section{8}

11386917

Sanger Institute

\section{6}

TIGR

Sanger Institute

Sanger Institute

Sanger Institute

Sanger Institute

$\mathbf{1 7 5 3 8 4 9 4}$

1753849

21106027

7509275

$\underline{17551082}$

7594741

$\underline{11258525}$

7271000

TIGR

22774026

1655576

1655578

1655580

16124306

DOE Joint Institute

21646663

22971187

1808591

23586111

Sanger Institute

$\underline{15896110}$

Sanger Institute

18311159

$\underline{\mathbf{2 8 2 0 2 5 1 7}}$

TIGR

TIGR

TIGR

23493563

19552815

TIGR

TIGR

TIGR

23137174

28278852

TIGR

DOE Joint Institute

TIGR

$\mathbf{1 5 8 0 5 6 6 7}$ 
Desulfovibrio desulfuricans

Desulfovibrio vulgaris

Dianthus caryophyllus

Dichelobacter nodosus

Dictyostelium discoideum

Drosophila melanogaster

Drosophila pseudoobscura

Ehrlichia chaffensis

Ehrlichia ruminantium

Elaeagnus umbellata 1

Elaeagnus umbellata 2

Enterococcus faecalis

Escherichia coli (metK)

Escherichia coli (metX)

Fibrobacter succinogenes

Fusarium sporotrichioides

Fusobacterium nucleatum

Gallus gallus

Gemmata obscuriglobus

Giardia lamblia

Glycine max 1

Glycine max 2

Glycine max 3

Gossypium hirsutum 1

Gossypium hirsutum 2

Gossypium hirsutum 3

Haemophilus influenzae

Haemophilus somnus

Helicobacter pylori

Helicobacter pylori 599

Heliobacillus mobilis

Homo sapiens 1

Homo sapiens 2

Hordeum vulgare 1

Hordeum vulgare 2

Hordeum vulgare 3

Ictalurus punctatus

Klebsiella pneumoniae

Lactobacillus gasseri

Lactobacillus plantarum

Lactococcus lactis

Lactuca sativa 1

Lactuca sativa 2

Legionella pneumophila

Leishmania infantum

Leptospira interrogans

Leuconostoc mesenteroides
23475965

TIGR

7434012

TIGR

Sanger Institute

$\underline{7296263}$

Baylor

TIGR

Sanger Institute

13540316

13540318

$\underline{29342835}$

1708999

$\underline{\mathbf{2 6 2 5 0 3 6 7}}$

TIGR

Univ. Oklahoma

19703697

TIGR

TIGR

29247850

TIGR

TIGR

TIGR

TIGR

TIGR

TIGR

1170942

$\underline{23467639}$

3024119

6685665

$\underline{27262362}$

4557737

284394

7434000

TIGR

TIGR

TIGR

Univ. Washington

DOE Joint Institute

$\mathbf{2 8 3 7 8 0 5 7}$

$\mathbf{1 3 8 7 8 5 7 6}$

TIGR

TIGR

Columbia

20387266

24215333

23023832

$1609 n / 1$ 
Listeria monocytogenes

Litchi chinensis

Lotus japonicus 1

Lotus japonicus 2

Lycopersicon esculentum 1

Lycopersicon esculentum 2

Lycopersicon esculentum 3

Lycopersicon esculentum 4

Magnaporthe grisea

Magnetococcus sp. MC-1

Magnetospirillum magnetotacticum

Mannheimia haemolytica

Medicago truncatula 1

Medicago truncatula 2

Medicago truncatula 3

Medicago truncatula 4

Mesembryanthemum crystallinum

Mesorhizobium loti 1

Mesorhizobium loti 2

Methylobacterium extorquens

Methylococcus capsulatus

Microbulbifer degradans

Moraxella catarrhalis

Mus musculus 1

Mus musculus 2

Musa acuminata

Mycobacterium avium

Mycobacterium bovis

Mycobacterium leprae

Mycobacterium marinum

Mycobacterium smegmatis

Mycobacterium tuberculosis

Mycoplasma genitalium

Mycoplasma penetrans

Mycoplasma pneumoniae

Mycoplasma pulmonis

Myxococcus xanthus

Neisseria gonorrhoeae

Neisseria meningitidis

Neorickettsia sennetsu

Neurospora crassa

Nicotiana tabacum

Nitrosomonas europaea

Nostoc sp. PCC 71201

Nostoc sp. PCC 71202

Novosphingobium aromaticivorans

Oceanobacillus iheyensis
16411100

30142157

21907982

TIGR

$\underline{1084406}$

$\underline{481566}$

1084408

TIGR

TIGR

23000957

$\underline{23015399}$

Baylor

TIGR

TIGR

TIGR

TIGR

1724104

20803994

13475107

Univ. Washington

TIGR

$\underline{23027148}$

DDJB Japan

476917

13097429

2305014

TIGR

Sanger Institute

15214074

Sanger Institute

TIGR

3915763

1346527

$\underline{\mathbf{2 6 5 5 3 5 4 7}}$

2500686

15829173

27804841

Univ. Oklahoma

$\mathbf{1 1 2 5 8 5 1 6}$

TIGR

2133316

7230379

$\underline{22954639}$

17231616

17132339

$\underline{23110658}$

22777999

22020264 
Oryza sativa 1

Oryza sativa 2

Oryza sativa 3

Pasteurella multocida

Pectobacterium carotovorum

Pectobacterium chrysanthemi

Petunia $x$ hybrida 1

Petunia $x$ hybrida 2

Phanerochaete chrysosporium

Phaseolus lunatus

Photorhabdus asymbiotica

Phytophthora infestans

Pinus banksiana

Pinus contorta 1

Pinus contorta 2

Pinus contorta 3

Pinus contorta 4

Pinus contorta 5

Pisum sativum

Plasmodium berghei

Plasmodium chabaudi

Plasmodium falciparum

Plasmodium knowlesi

Plasmodium yoelii

Populus deltoides

Porphyromonas gingivalis

Prevotella intermedia

Prochlorococcus marinus 1

Prochlorococcus marinus 2

Pseudomonas aeruginosa

Pseudomonas fluorescens

Pseudomonas putida

Pseudomonas syringae

Psychrobacter sp.273-4

Ralstonia eutropha

Ralstonia metallidurans

Ralstonia solanacearum

Rattus norvegicus 1

Rattus norvegicus 2

Rhizobium leguminosarum

Rhodobacter sphaeroides

Rhodopseudomonas palustris

Rhodospirillum rubrum

Rickettsia prowazekii

Rickettsia typhi

Saccharomyces cerevisiae 1

Saccharomyces cerevisiae 2

Crlmanalla tambi
450549

$\underline{\mathbf{1 7 7 8 8 2 1}}$

8468037

$\underline{13431697}$

Sanger Institute

TIGR

$\underline{1084428}$

$\mathbf{5 7 2 6 5 9 4}$

DOE Joint Institute

$\underline{18157331}$

Sanger Institute

$\underline{23394401}$

$\underline{1033190}$

$\underline{10441429}$

$\underline{10441431}$

TIGR

TIGR

TIGR

\section{9}

Sanger Institute

Sanger Institute

10129955

Sanger Institute

23482440

497900

TIGR

TIGR

$\underline{23132136}$

$\underline{23122176}$

$\underline{15595743}$

$\underline{23060543}$

$\underline{26991645}$

$\underline{23471420}$

DOE Joint Institute

DOE Joint Institute

$\underline{22979451}$

$\underline{17544853}$

92483

19705457

Sanger Institute

22957691

$\underline{22961217}$

22965557

7387879

$\underline{11133588}$

$\underline{1346525}$

83324

16766201 
Schistosoma japonicum

Schizosaccharomyces pombe

Secale cereale

Serratia marcescens

Shewanella oneidensis

Shigella flexneri

Silicibacter pomeroyi

Sinorhizobium meliloti

Solanum tuberosum 1

Solanum tuberosum 2

Solanum tuberosum 3

Sorghum bicolor

Spiroplasma kunkelii

Staphylococcus aureus

Staphylococcus epiderminis

Streptococcus agalactiae

Streptococcus equi

Streptococcus gordonii

Streptococcus mitis

Streptococcus mutans

Streptococcus pneumoniae

Streptococcus pyogenes

Streptococcus sobrinus

Streptococcus suis

Streptococcus thermophilus

Streptococcus uberis

Streptomyces avermitilis

Streptomyces coelicolor

Streptomyces fradiae

Streptomyces pristinaespiralis

Streptomyces spectabilis

Suaeda maritima

Synechococcus sp.

Synechocystis sp.

Takifugu rubripes 1

Takifugu rubripes 2

Tetraodon nigroviridis

Thalassiosira pseudonana

Theileria annulata

Theileria parva

Thermobifida fusca

Thermosynechococcus elongatus

Thermotoga maritima

Treponema pallidum

Trichodesmium erythraeum

Triticum aestivum 1

Triticum aestivum 2
EBI Parasites

$\underline{\mathbf{7 4 9 3 3 5 2}}$

TIGR

Sanger Institute

$\underline{24372516}$

$\mathbf{2 4 1 1 4 1 9 7}$

TIGR

15964164

TIGR

TIGR

TIGR

TIGR

Univ. Oklahoma

$\underline{1709003}$

9624212

29611810

Sanger Institute

TIGR

TIGR

29611808

15902715

19746332

TIGR

Sanger Institute

Univ. C. Louvain

Sanger Institute

29833416

21219978

$\underline{15554326}$

2294502

7387884

$\underline{11992267}$

$\underline{23134392}$

7434007

DOE Joint Institute

DOE Joint Institute

Genoscope

DOE Joint Institute

Sanger Institute

TIGR

23017995

29611806

7387883

15639781

$\underline{23040652}$

TIGR

TIGR

TISD 
Triticum aestivum 4

Tropheryma whipplei

Trypanosoma brucei

Trypanosoma cruzi

Ureaplasma urealyticum

Vibrio cholerae

Vibrio parahaemolyticus

Vibrio vulnificus

Wigglesworthia brevipalpis

Wolbachia sp.

$X$-bacteria

Xanthomonas axonopodis

Xanthomonas campestris

Xenopus laevis 1

Xenopus laevis 2

Xenopus tropicalis

Xylella fastidiosa

Yersinia enterocolitica

Yersinia pestis

Zea mays 1

Zea mays 2
TIGR

28572562

TIGR

Sanger Institute

$\underline{13357974}$

9654898

28899380

27364907

24324056

TIGR

Direct submission

21241583

$\underline{21230235}$

TIGR

27882050

Sanger Institute

$\mathbf{1 5 8 3 6 9 9 4}$

Sanger Institute

16121235

TIGR

TIGR 
Table 2. Specific structural features shown by each group on the MAT phylogenetic tree.

\begin{tabular}{|c|c|c|c|}
\hline & \multicolumn{3}{|c|}{ The loop connecting $\beta$-strands A2-A3 has 10 residues } \\
\hline & \multirow{2}{*}{ Plants } & \multicolumn{2}{|c|}{$\begin{array}{l}\text { Presence of the sequences }{ }^{93} \text { FXSXDVXLXAD }{ }^{103},{ }^{216} \text { NDEIA }^{220} \text {, } \\
\text { and }{ }^{331} \text { VFVD }^{334}\end{array}$} \\
\hline & & \multicolumn{2}{|c|}{$\begin{array}{l}\text { Insertion of 3-4 residues in the loop connecting helixes } 7 \text { and } 8 \text {, } \\
\text { between positions } 369-370\end{array}$} \\
\hline & \multirow{3}{*}{ Vertebrates } & Fishes & $\begin{array}{l}\text { Present specific residues for the MAT I enzyme, } \\
\text { C69, C377 and V262 }\end{array}$ \\
\hline & & Birds/reptiles & $\begin{array}{l}\text { Present additional specific residues such as R82 } \\
\text { and D167, besides those shown in fishes }\end{array}$ \\
\hline & & Mammals & $\begin{array}{l}\text { Present a typical cysteine in the MAT I flexible } \\
\text { loop, C121 besides the residues specific for } \\
\text { fishes, birds and reptiles }\end{array}$ \\
\hline & \multicolumn{3}{|c|}{ Deletion of 3-4 residues in the loop connecting $\beta$-strands A2-A3 } \\
\hline & \multirow{3}{*}{ Proteobacteria } & \multicolumn{2}{|c|}{$\begin{array}{l}\text { Deletion of } 2 \text { residues in the loop connecting helix } 2 \text { and } \beta \text {-strand } \\
\text { B3 }\end{array}$} \\
\hline & & \multicolumn{2}{|c|}{ Insertion of 4 residues between helix 4 and $\beta$-strand $A 4$} \\
\hline & & \multicolumn{2}{|c|}{ Isertion of 4-5 residues between helixes 8-9 } \\
\hline & \multirow{4}{*}{ Cyanobacteria } & \multicolumn{2}{|c|}{ Insertion of 4 residues in the loop between $\beta$-strand B3 and helix 4} \\
\hline & & \multicolumn{2}{|c|}{$\begin{array}{l}\text { Insertion of 6-7 residues in the loop connecting helix } 4 \text { and } \beta \text { - } \\
\text { strand A4 }\end{array}$} \\
\hline & & \multicolumn{2}{|c|}{ Insertion of 7 residues in the loop between helixes 7 and 8} \\
\hline & & \multicolumn{2}{|c|}{ Insertion of 2 residues in the loop connecting helixes 8 and 9} \\
\hline & \multirow{4}{*}{ CFB group } & \multicolumn{2}{|c|}{$\begin{array}{l}\text { Insertion of } 1-2 \text { residues in the loop connecting helix } 3 \text { and } \beta \text { - } \\
\text { strand A2 }\end{array}$} \\
\hline & & \multicolumn{2}{|c|}{$\begin{array}{l}\text { Insertion of } 2 \text { residues in the loop connecting } \beta \text {-strand } C 3 \text { and } \\
\text { helix } 6\end{array}$} \\
\hline & & \multicolumn{2}{|c|}{$\begin{array}{l}\text { Insertion of 9-11 residues in the loop connecting helix } 4 \text { and } \beta \text { - } \\
\text { strand A4 }\end{array}$} \\
\hline & & \multicolumn{2}{|c|}{ Insertion of 21-22 residues in the loop connecting helixes 8 and 9} \\
\hline & \multirow[b]{2}{*}{ Actinobacteria } & \multicolumn{2}{|c|}{ Insertion of 8 residues in the flexible loop } \\
\hline & & \multicolumn{2}{|c|}{$\begin{array}{l}\text { Insertion of 2-4 residues in the loop connecting helix } 4 \text { and } \beta \text { - } \\
\text { strand A4 }\end{array}$} \\
\hline & Firmicutes & Mollicutes & $\begin{array}{l}\text { Deletion of } 7 \text { residues in the loop connecting } \\
\text { helix } 2 \text { and } \beta \text {-strand B3 }\end{array}$ \\
\hline
\end{tabular}


sec. structure Identity

Rat liver HAT

sec. structure Identity Rat liver HAT

sec. structure Identity Rat liver_MAT

Sec. structure Identity

Rat liver MAT

sec. structure

Identity

Rat liver ндт

Sec. structure

Identity

Rat liver идт

sec. structure Identity Rat liver HAT

sec. structure Ident1ty Rat I1Ver MAT

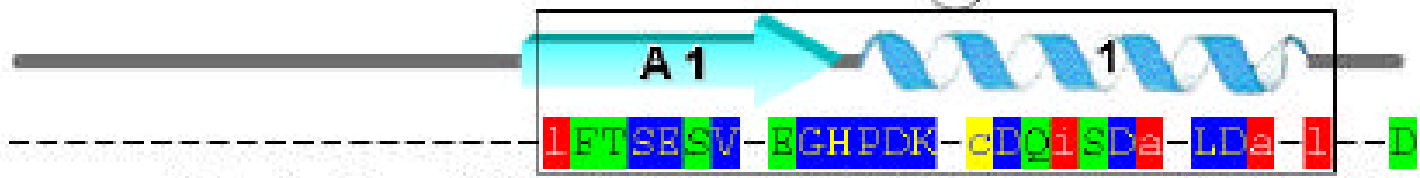
mngp vdgl cdhs l seeGAFMETSESVGEGHPDKICDQI SDAVLDAHLKQD

(II)

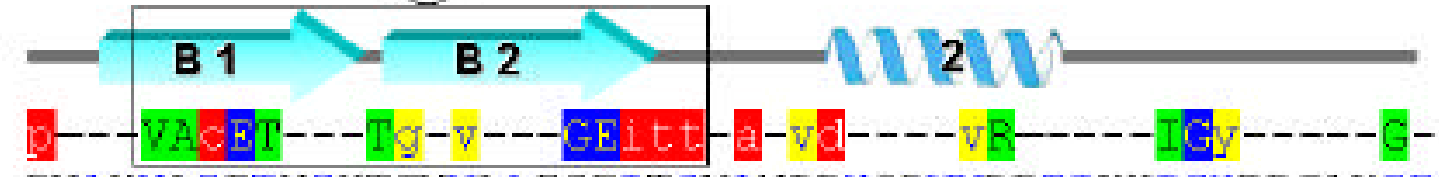
PNAKWACETVCKTGMVLCGEITSMAMI DYQRVVRDT IKHIGYDDSAKGE

(III)

(III)

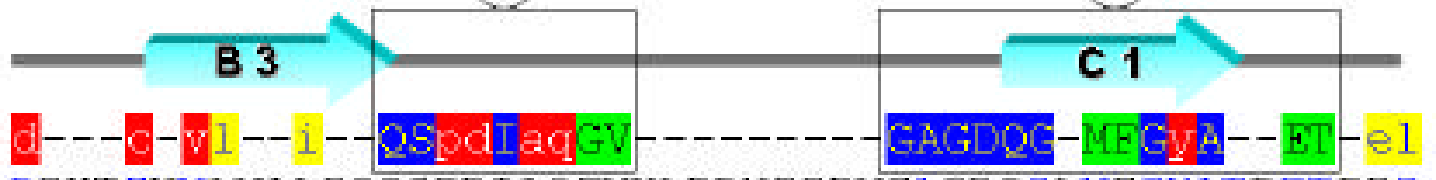

DEKTCNVLVALEOOSEDIAOCVHLDRNEEDVGAGDOGLWFGYATDETEEC 15!

(IV)

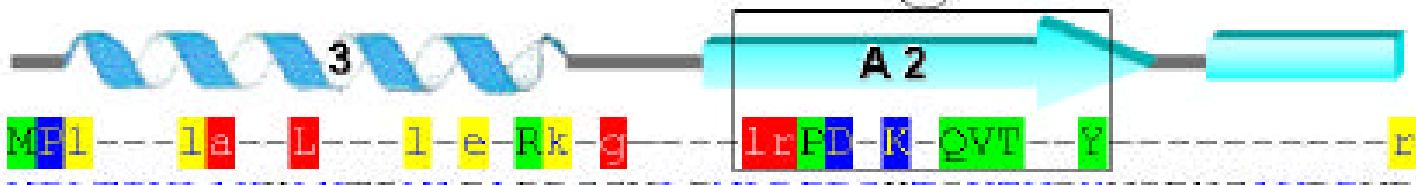

MPLTIVLAHKLNTRMADLRRSGVLPWRFDSKTQVTVQYVQDNGAVIPVR 201

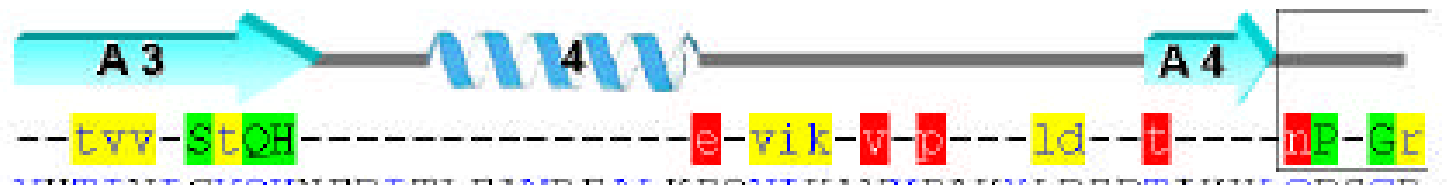

VHTIVISWQHNEDITLEANREALKEQVIKAVVPAKY LDEDTIYHLQRSGR 25।

(v)

\section{5}

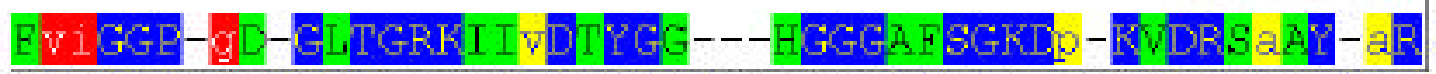
EVIGGPQGDAGVTGRII IVDTYGGWGAHGGGAF SGKDYTKVDRSAAYAAR 306

(vi)

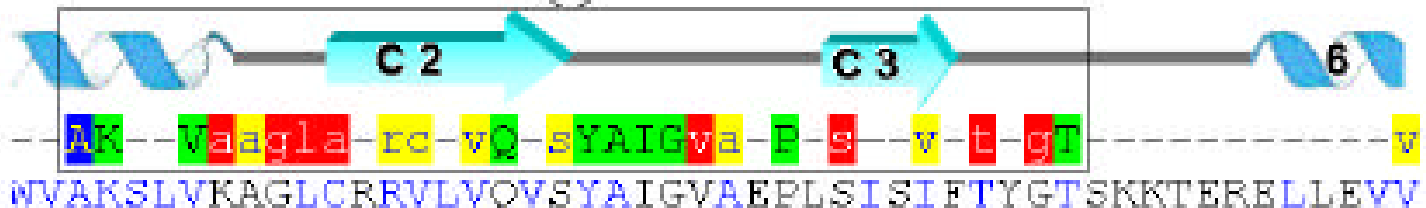

(VII)

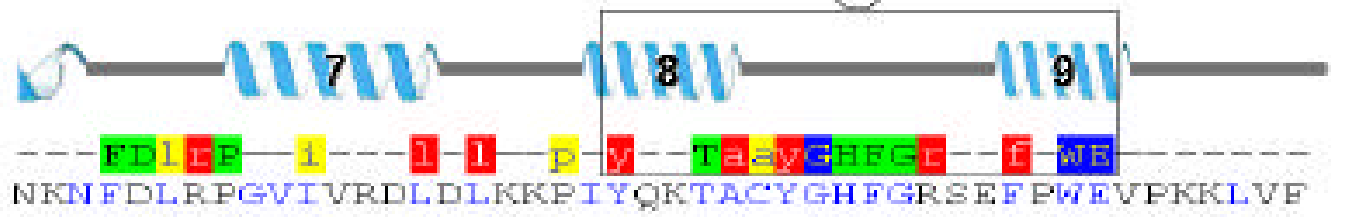




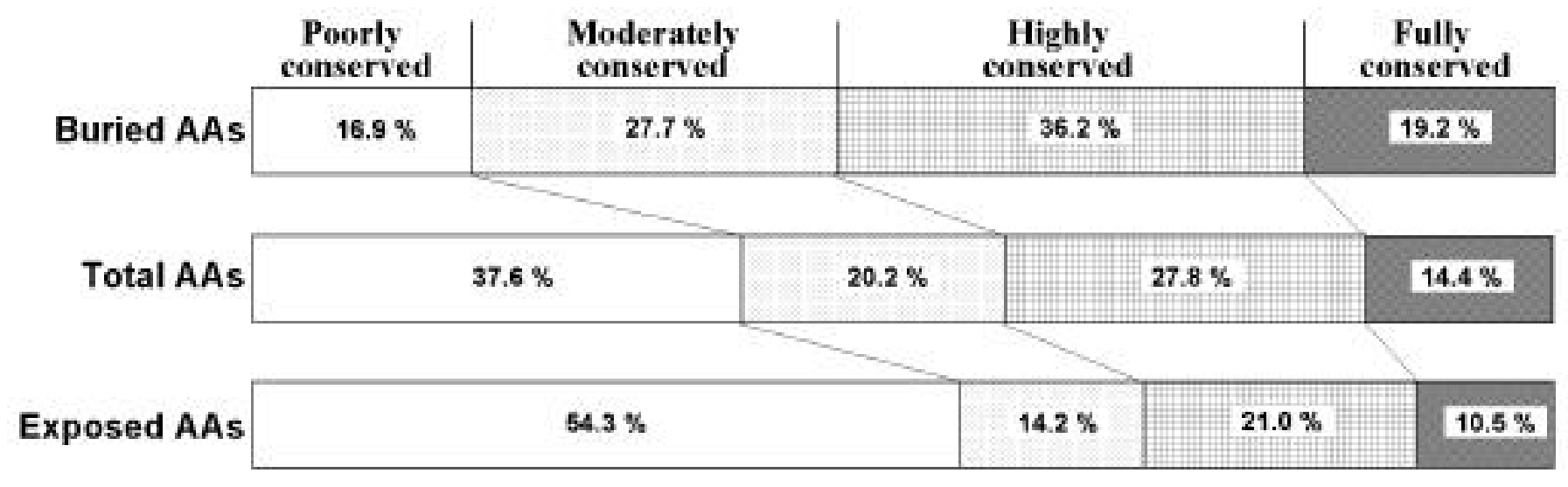




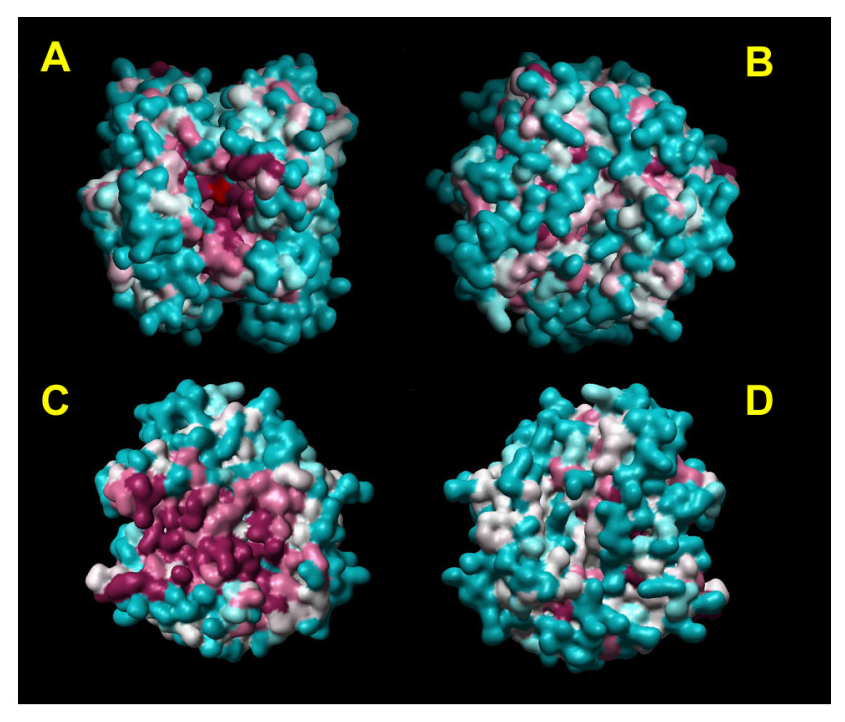




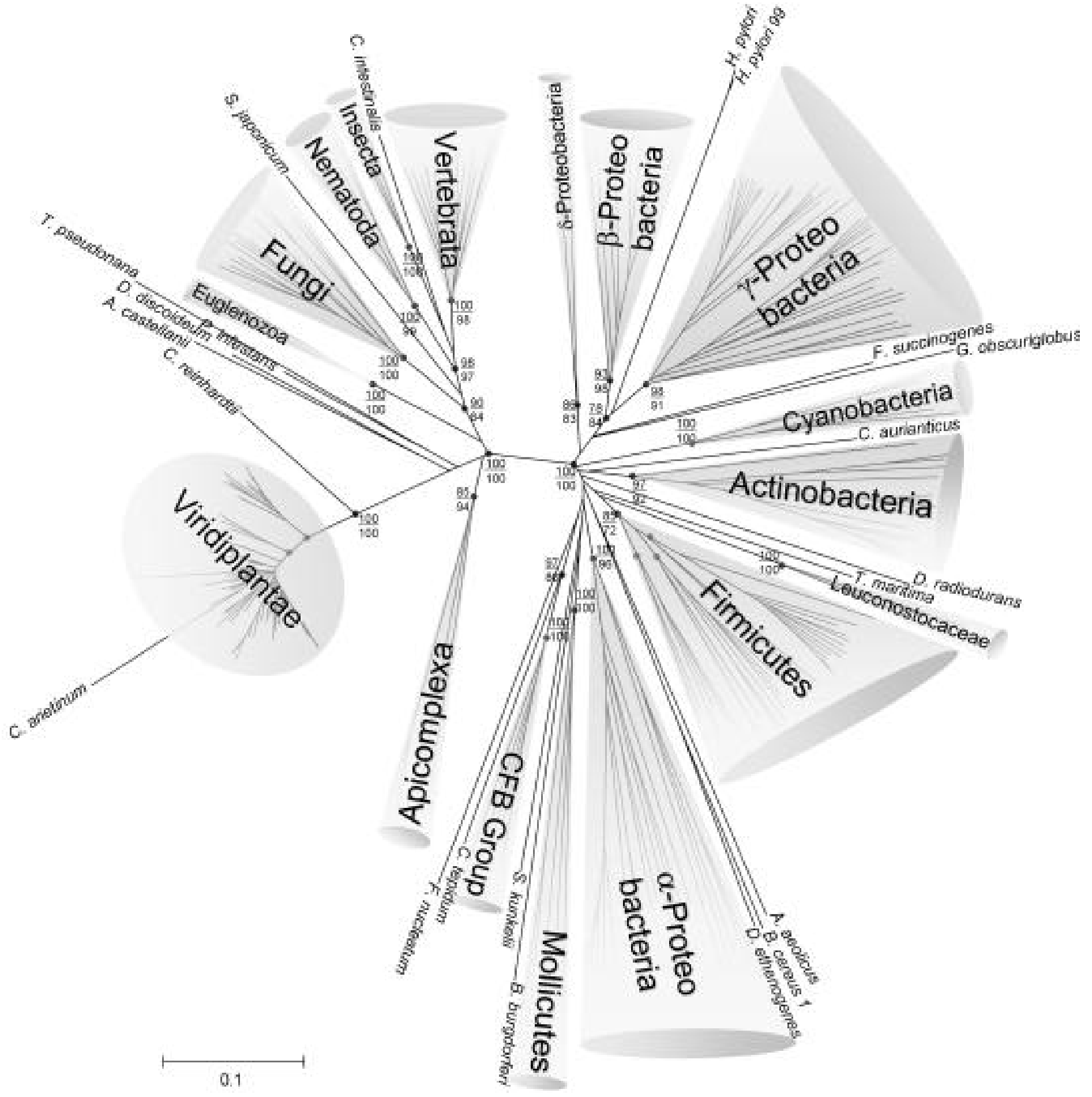




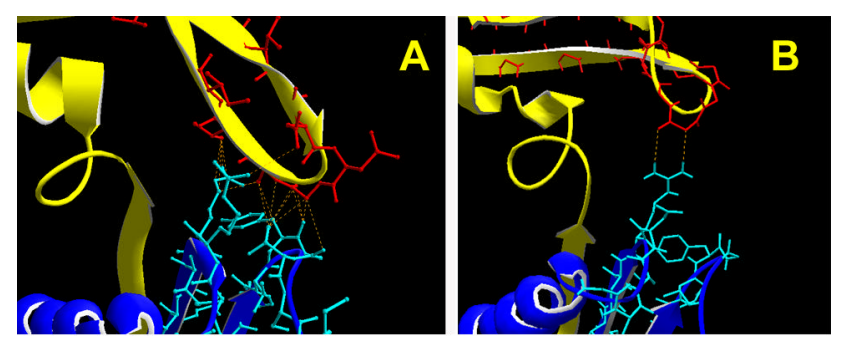

